\title{
Second-site mutation in the Wiskott-Aldrich syndrome (WAS) protein gene causes somatic mosaicism in two WAS siblings
}

\author{
Taizo Wada, ${ }^{1}$ Akihiro Konno, ${ }^{1}$ Shepherd H. Schurman, ${ }^{1}$ Elizabeth K. Garabedian, ${ }^{2}$ \\ Stacie M. Anderson, ${ }^{1}$ Martha Kirby, ${ }^{1}$ David L. Nelson, ${ }^{3}$ and Fabio Candotti ${ }^{1}$
}

${ }^{1}$ Genetics and Molecular Biology Branch,

${ }^{2}$ Medical Genetics Branch, National Human Genome Research Institute, and

${ }^{3}$ Metabolism Branch, National Cancer Institute, National Institutes of Health, Bethesda, Maryland, USA

\begin{abstract}
Revertant mosaicism due to true back mutations or second-site mutations has been identified in several inherited disorders. The occurrence of revertants is considered rare, and the underlying genetic mechanisms remain mostly unknown. Here we describe somatic mosaicism in two brothers affected with Wiskott-Aldrich syndrome (WAS). The original mutation causing disease in this family is a single base insertion (1305insG) in the WAS protein (WASP) gene, which results in frameshift and abrogates protein expression. Both patients, however, showed expression of WASP in a fraction of their T cells that were demonstrated to carry a second-site mutation causing the deletion of 19 nucleotides from nucleotide 1299 to 1316 . This deletion abrogated the effects of the original mutation and restored the WASP reading frame. In vitro expression studies indicated that mutant protein encoded by the second-site mutation was expressed and functional, since it was able to bind to cellular partners and mediate $\mathrm{T}$ cell receptor/CD3 downregulation. These observations were consistent with evidence of in vivo selective advantage of WASP-expressing lymphocytes. Molecular analysis revealed that the sequence surrounding the deletion contained two 4-bp direct repeats and that a hairpin structure could be formed by five GC pairs within the deleted fragment. These findings strongly suggest that slipped mispairing was the cause of this second-site mutation and that selective accumulation of WASP-expressing T lymphocytes led to revertant mosaicism in these patients.
\end{abstract}

J. Clin. Invest. 111:1389-1397 (2003). doi:10.1172/JCI200315485.

\section{Introduction}

The Wiskott-Aldrich syndrome (WAS) is an X-linked disorder that is characterized by thrombocytopenia, eczema, impaired cellular and humoral immunity, and increased susceptibility to lymphoid malignancies and autoimmunity (1). The gene responsible for WAS was identified by positional cloning and encodes the WAS protein (WASP), a 502-amino acid proline-rich peptide (2). WASP is constitutively expressed in cytoplasm of all nonerythroid

Received for publication March 19, 2002, and accepted in revised form February 4, 2003.

Address correspondence to: Fabio Candotti, Disorders of Immunity Section, Genetics and Molecular Biology Branch, National Human Genome Research Institute, National Institutes of Health, 49 Convent Drive, Building 49, Room 3A20, MSC 4442, Bethesda, Maryland 20892-4442, USA.

Phone: (301) 435-2944; Fax: (301) 480-3678;

E-mail: fabio@nhgri.nih.gov.

Taizo Wada's present address is: Department of Pediatrics, Kanazawa University Graduate School of Medical Science, Kanazawa, Japan.

Taizo Wada and Akihiro Konno contributed equally to this work. Conflict of interest: The authors have declared that no conflict of interest exists.

Nonstandard abbreviations used: Wiskott-Aldrich syndrome (WAS); recombinant IL-2 (rIL-2); polymorphonuclear cell (PMN); phycoerythrin (PE); glutathione S-transferase (GST); T cell receptor- $\beta$ (TCR- $\beta$ ); verprolin homology, cofilin homology, and acidic (VCA). hematopoietic cells $(3,4)$ and appears to be of central importance for signal transduction and cytoskeleton reorganization events of hematopoietic cells (5).

Somatic revertant mosaicism has been described in an increasing number of genetic disorders. Both back mutations leading to restoration of wild-type sequences (6-15) and second-site mutations resulting in compensatory changes (16-18) have been demonstrated in mosaic individuals. Revertant mosaicism acquires a particular clinical relevance in the case of patients affected with a primary immunodeficiency because it may lead to selective growth advantage of the corrected cells and improvement of disease symptoms. In fact, spontaneous in vivo reversion has been reported in single cases of adenosine deaminase deficiency (12), X-linked severe combined immunodeficiency (8), and WAS (15), where patients showed atypical and/or progressively mild clinical course because of the selective growth advantage of the revertant lymphocytes. To date, evidence has been provided for at least three mechanisms leading to back mutations or secondsite mutations in inherited disorders: intragenic recombination $(7,10)$, gene conversion (9), and DNA polymerase slippage $(11,15,18)$. Slippage-type events are thought to cause insertion and deletion mutations of small repetitive sequences in a number of human genetic diseases and explain the instability of repetitive sequences observed in bacterial, yeast, and eukaryotic sys- 
tems (19-22). Polymerase slippage is also the most commonly invoked mechanism to explain triplet repeat expansion in human diseases (e.g., Huntington disease, fragile X syndrome, and Friedreich ataxia) (23).

Here, we described two WAS siblings with revertant mosaicism due to the same second-site mutation, likely caused by slipped mispairing. The original mutation responsible for the disease in this family is single base $G$ insertion predicted to cause frameshift and premature termination of the protein. A second-site mutation was found in $\mathrm{T}$ lymphocytes from both brothers that carried a deletion of a $19 \mathrm{bp}$ fragment, including the original mutation. This deletion resulted in restoration of the reading frame and the generation of an internally truncated, yet functional, WASP protein. Revertant T lymphocytes showed WASP expression and selective growth advantage in vivo over the WASP-negative (WASP-) cells. The occurrence of the same second-site mutation in two subjects from the same family strongly suggests a common underlying mechanism, and the characteristics of nucleotide sequence involved in the mutation are clearly compatible with slipped strand mispairing. From our observations we hypothesize that slipped mispairing is commonly occurring in the contest of this particular sequence, but only recognized in the rare cases in which selective advantage permits the accumulation of revertant cells above the detection threshold.

\section{Methods}

Case presentation. Figure 1 shows the pedigree of the patients' family. Patient II-1 is an 18-year-old male who developed petechiae early after birth when his platelet count was in the range $7,000-20,000 / \mu 1$. In the first year of life, he had eczema, otitis media, and conjunctivitis. After a clinical diagnosis of WAS was made at the age of 16 months, he underwent a splenectomy because of persistent thrombocytopenia. Between the age of 2 and 3 years, he had multiple episodes of pneumonia and recurrent leukocytoclastic vasculitis. Frequent ear infections and one episode of bronchitis are described until the age of 6 , when he suffered from streptococcal pneumonia and sepsis. Between the ages of 12 and 16 years, he had documented episodes of medium vessel vasculitis leading to recurrent skin ulcers.

Patient II-1's younger brother (II-2) also developed thrombocytopenia early after birth and underwent a splenectomy at the age of 6 months. The postoperative clinical history is remarkable for occasional otitis media and continued severe eczema until the age of 9 years, when he developed molluscum contagiosum, which progressed unremittingly for 3 years until all extremities and truncal surfaces accessible to hands were affected. At age 13, he developed non-Hodgkin B cell lymphoma, which relapsed after chemotherapy at the age of 15 . Both clinical histories are consistent with severe WAS phenotype (score of 5) (24). No clinical improvement with age has yet been observed in either patient. No history of other affected males was noted in the maternal side of the family.
Cell preparation. PBMCs were isolated by FicollHypaque (Mediatech Inc., Washington, DC, USA) gradient centrifugation from the patients and healthy controls. For T lymphocyte cultures, PBMCs were maintained in the presence of $100 \mathrm{ng} / \mathrm{ml}$ of anti-CD3 (OKT3; Ortho Diagnostic Systems Inc., Raritan, New Jersey, USA), $5 \mu \mathrm{g} / \mathrm{ml}$ of anti-CD28 mAb (PharMingen, San Diego, California, USA), and $100 \mathrm{IU} / \mathrm{ml}$ of recombinant IL-2 (rIL-2) (gift of C. Reynolds, National Cancer Institute, Frederick, Maryland, USA) for 3 days in RPMI-1640 medium (Life Technologies Inc., Rockville, Maryland, USA) containing 10\% FBS (Gemini BioProducts, Woodland, California, USA) and antibiotics. Cells were then cultured in RPMI-1640 plus $10 \%$ FBS medium supplemented with $100 \mathrm{IU} / \mathrm{ml}$ of rIL-2 for 12 days. Human T lymphotropic virus type 1-transformed WAS T cell lines carrying the $57 \mathrm{delG}$ "null" mutation (25) were maintained in the same medium. MOLT-4 cells were obtained from American Type Culture Collection (Manassas, Virginia, USA).

Flow-cytometric analysis of WASP and cell sorting. Flow cytometric analysis of WASP was performed as described previously (15) with minor modifications. PBMCs were stained for cell surface antigens prior to cell membrane permeabilization using the following mAb's: CyChromeconjugated anti-CD3 or anti-CD20 (PharMingen); TRIcolor-labeled anti-CD8 (Caltag Laboratories Inc., Burlingame, California, USA); FITC-conjugated antiCD4 (Caltag Laboratories Inc.); FITC-conjugated antiCD45RA and anti-CD45R0 (PharMingen); and FITCconjugated anti-CD14 or anti-CD56 (Becton Dickinson Immunocytometry Systems, San Jose, California, USA). After washing, cells were fixed and permeabilized with CytoStain kit (PharMingen) and were incubated with 1:200 diluted anti-WASP (3F3-A5) mAb (3) or purified mouse IgG- 1 (PharMingen) at $4^{\circ} \mathrm{C}$ for $20 \mathrm{~min}$. Cells were then reacted with biotin-conjugated anti-mouse IgG-1 at $4^{\circ} \mathrm{C}$ for $20 \mathrm{~min}$, followed by further incubation with phycoerythrin-conjugated (PE-conjugated) streptavidin (both from PharMingen). Stained cells were analyzed with a FACScan flow cytometer and the CellQuest software (Becton Dickinson Immunocytometry Systems).

For sequencing analysis, WASP-expressing (WASP $\left.{ }^{+}\right)$ and WASP- ${ }^{-}$cells were purified from cultured T lymphocytes using a FACSVantage cell sorter (Becton Dickinson Immunocytometry Systems). The purity of

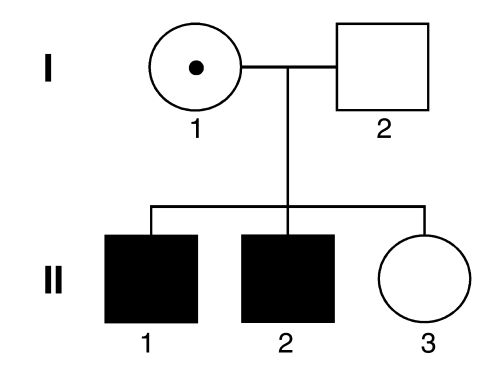

Figure 1

Pedigree of the study family. Solid symbols represent affected individuals, and the carrier status of subject I- 1 is indicated by the central dot. 
sorted $\mathrm{WASP}^{+}$and WASP- $\mathrm{T}$ lymphocytes was more than $98 \%$, as determined by a flow cytometric analysis. For mutation detection in lineage-specific cells using fluorescent genotyping, PBMCs and polymorphonuclear cells (PMNs) were separated by Ficoll-Hypaque gradient centrifugation. $\mathrm{CD}^{+} \mathrm{T}$ cells were isolated from PBMCs and PMNs with immunomagnetic beads (Dynal Inc., Lake Success, New York, USA), following manufacturer's instructions. CD3-depleted PMNs were frozen and stored until use. The CD3-negative PBMC fraction was divided in two aliquots that were separately stained with PE-conjugated anti-CD14, CyChrome-conjugated anti-CD20 or PE-conjugated anti-CD20, or CyChromeconjugated anti-CD56 (all from PharMingen). Both aliquots were also stained with FITC-conjugated anti-TCR- $\alpha \beta$ and anti-TCR- $\gamma \delta$ (both from Becton Dickinson Immunocytometry Systems) for detection of residual T lymphocytes. CD $14^{+} /$TCR- $\alpha \beta^{-} /$TCR- $\gamma \delta^{-}, \mathrm{CD} 20^{+} /$ TCR- $\alpha \beta^{-} /$TCR- $\gamma \delta^{-}$, and CD $56^{+} /$TCR- $\alpha \beta^{-} /$TCR- $\gamma \delta^{-}$cells were then isolated using a FACSVantage cell sorter (Becton Dickinson Immunocytometry Systems).

Western blot analysis and in vitro binding assay. Western blot analysis of WASP and in vitro binding assay were performed as described $(25,26)$. Briefly, for Western blot analysis, cell lysates $(10-100 \mu \mathrm{g})$ were boiled, subjected to $8 \%$ SDS-PAGE, and electrotransferred onto nylon membranes (Immobilon-P; Millipore Corp., Bradford, Massachusetts, USA). For the in vitro binding assay, 100 $\mu \mathrm{g}$ of cell lysate protein was incubated with $5 \mu \mathrm{g}$ of Fyn-Src homology 3 (SH3) glutathione S-transferase (GST) fusion protein bound to agarose beads (Santa Cruz Biotechnology Inc., Santa Cruz, California, USA) and Grb2-GST agarose (Upstate Biotechnology Inc., Lake Placid, New York, USA) in $500 \mu$ of lysis buffer (300 mM NaCl, $50 \mathrm{mM}$ Tris-HCl, $2 \mathrm{mM}$ EDTA, 0.5\% Triton X-100, $2.5 \mathrm{mM} p$-nitrophenyl $p^{\prime}$-guanidino-benzoate, and $10 \mu \mathrm{g} / \mathrm{ml}$ aprotinin and leupeptin) for $2 \mathrm{~h}$ at $4^{\circ} \mathrm{C}$. Immunoprecipitates were then washed, boiled, and resolved using $12 \%$ SDS-PAGE. Detection of WASP was performed using the anti-WASP (3F3-A5), or B-9 (Santa Cruz Biotechnology Inc.) mAb's, a rabbit polyclonal $\mathrm{Ab}$ purchased from Upstate Biotechnology Inc. A rabbit polyclonal Ab specific for STAT5a was used for reprobing of membranes as described previously (27). DNA extraction and mutation analysis of the WASP gene. DNA was extracted from blood samples and cell lines using the QIAamp DNA blood mini kit (QIAGEN Inc., Valencia, California, USA). Each exon of the WASP gene was amplified from genomic DNA using specific primers (15). Sequencing was performed on gel-purified PCR products or PCR products subcloned in the pCR2.1 vector (Invitrogen Corp., Carlsbad, California, USA) using the ABI Prism BigDye Terminator Cycle sequencing kit on an ABI Prism 310 Genetic Analyzer (Perkin-Elmer Applied Biosystems, Foster City, California, USA), as described previously (15).

For fluorescent genotyping (GeneScan) analysis, the nucleotide 1150-1358 fragment of the WASP genomic DNA was amplified from $300 \mathrm{ng}$ of DNA extracted from PBMCs, immunomagnetic bead-purified $\mathrm{CD}^{+} \mathrm{T}$ lymphocytes, $\mathrm{CD}^{+}$cell-depleted PMNs and sorted $\mathrm{CD} 20^{+} \mathrm{B}$ lymphocytes, CD56 $6^{+} \mathrm{NK}$ cells, and CD $14^{+}$ monocytes, using 20 pmol each of the $5^{\prime}$-TCC AGC TAC TGG ACG TTC TG-3' forward primer and the FAMlabeled 5'-TTC CCT GCC GGA TTT GAT-3' reverse primer in a $50-\mu \mathrm{l}$ volume reaction containing $1.25 \mathrm{U}$ Ex Taq polymerase, $1 \times$ Ex Taq Buffer, $2 \mathrm{mM} \mathrm{Mg}^{2+}$, and $200 \mu \mathrm{M}$ dNTPs (all from Takara Bio Inc., Shiga, Japan). Thirtyfive amplification cycles $\left(30 \mathrm{~s}\right.$ at $94^{\circ} \mathrm{C}, 30 \mathrm{~s}$ at $60^{\circ} \mathrm{C}, 30 \mathrm{~s}$ at $72^{\circ} \mathrm{C}$ ) were carried out. One microliter of the amplification product was then combined to $12 \mu \mathrm{l}$ of deionized formamide and $0.5 \mu \mathrm{l}$ of GeneScan size standard (Perkin-Elmer Applied Biosystems) and analyzed on a ABI Prism 310 Genetic Analyzer using the GeneScan Analysis Software (Perkin-Elmer Applied Biosystems). Titration studies showed that this procedure allowed detection of one copy of the $\Delta 19 \mathrm{bp}$ mutation present within 5,000 cells (data not shown).

Analysis of T cell receptor $-\beta$ chain gene rearrangements. T cell receptor- $\beta$ (TCR- $\beta$ ) gene rearrangements were detected by family-specific PCR in combination with the GeneScan technique as described recently (28), with minor modifications. Briefly, 50 ng of DNA extracted from sorted WASP ${ }^{+}$and WASP- $T$ lymphocytes was subjected to PCR amplification using a TCR V $\beta$ consensus primer [V $\beta$ pan: $5^{\prime}$-CTC GAA TTC T(T/G) T(A/T) (C/T)T GGT A(C/T) C(G/A)(T/A)CA-3'; 30 pmol] and a TCR-J $\beta$ family-specific primer (J $\beta$ 2.1: 5'-ACG-GTG-AGC-CGT-GTC-CC-3'; 10 pmol) (28) in a 50- $\mu \mathrm{l}$ volume reaction containing $1.25 \mathrm{U}$ Ex Taq polymerase, $1 \times$ Ex Taq buffer, $2 \mathrm{mM} \mathrm{Mg}^{2+}$, and $200 \mu \mathrm{M}$ dNTP (all from Takara Bio Inc.). Thirty-five amplification cycles $\left(30 \mathrm{~s}\right.$ at $94^{\circ} \mathrm{C}, 40 \mathrm{~s}$ at $57^{\circ} \mathrm{C}, 40 \mathrm{~s}$ at $72^{\circ} \mathrm{C}$ ) were carried out. One microliter of the amplification product was then subjected to a seminested reaction using the same amplification conditions, but using a FAM-labeled $V \beta$ pan primer in combination with a nested J $\beta$ 2.1-specifc primer (5'-TGA-GCC-GTG-TCC-CTGGCC-CGA-A-3') (28). One microliter of the second PCR reaction product was combined with $12 \mu \mathrm{l}$ of deionized formamide and $0.5 \mu \mathrm{l}$ of GeneScan size standard (Perkin-Elmer Applied Biosystems) and analyzed on an ABI Prism 310 Genetic Analyzer using the GeneScan Analysis Software (Perkin-Elmer Applied Biosystems). DNA extracted from MOLT-4 cells with known clonal TCR-J $\beta 2.1$ rearrangement was used as positive control. WASP vector constructs and transductions. The GCWASP retroviral vector expressing the wild-type WASP and the neomycin resistance (Neo) genes and the transduction procedures used for gene transfer into WAS T cells have been described previously (25). Briefly, GCWASP viral particles pseudotyped with the gibbon ape leukemia virus envelope were produced using the PG13 cell line (29). To construct retroviral vectors carrying the patients' mutations, the exon 10 fragment of wild-type WASP was replaced at the XhoI and EcoRI sites with sequence-verified PCR products of the mutated exon 10 sequences amplified from patient II-1. The SAM-EN vector expressing only the neomycin resistance gene (30) was used as 
Table 1

Immunological features of patients II-1 and II-2

\begin{tabular}{|c|c|c|c|}
\hline & \multicolumn{2}{|c|}{ Patient } & \multirow[t]{2}{*}{ Normal range } \\
\hline & II-1 & $\mathrm{II}-2$ & \\
\hline \multicolumn{4}{|c|}{ Absolute blood count $(/ \mu \mathrm{l})$} \\
\hline WBCs & 6,890 & 4,850 & $4,500-11,000$ \\
\hline Lymphocytes & 703 & 601 & $1,500-3,000$ \\
\hline \multicolumn{4}{|c|}{ T lymphocyte subsets (\%) } \\
\hline $\mathrm{CD}^{+}$ & 58.8 & 59.0 & $60.9-84.0$ \\
\hline $\mathrm{CD}_{4}{ }^{+\mathrm{CD}_{3}}{ }^{+}$ & 42.9 & 49.9 & $32.0-57.9$ \\
\hline $\mathrm{CD}^{+}{ }^{\mathrm{CD}} 3^{+}$ & 11.9 & 4.7 & $10.9-36.2$ \\
\hline $\mathrm{CD}_{45 \mathrm{RA}^{+} \mathrm{CD} 4^{+}}$ & 13.1 & 1.2 & $3.6-22.4$ \\
\hline $\mathrm{CD}_{45 \mathrm{RO}^{+} \mathrm{CD}^{+}}{ }^{+}$ & 21.4 & 47.4 & $12.2-28.9$ \\
\hline $\mathrm{CD}_{45 \mathrm{RA}^{+} \mathrm{CD} 8^{+}}$ & 6.0 & 1.0 & $3.5-22.0$ \\
\hline $\mathrm{CD}_{45 \mathrm{R}^{+} \mathrm{CD}^{+}}{ }^{+}$ & 3.3 & 1.5 & $1.8-9.5$ \\
\hline 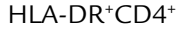 & 9.2 & 31.0 & $\leq 7.7$ \\
\hline $\mathrm{HLA}^{-\mathrm{DR}^{+} \mathrm{CD} 8^{+}}$ & 6.2 & 6.9 & $\leq 11.6$ \\
\hline
\end{tabular}

WBCs, white blood cells.

negative control. For transductions, WASP-null T cell lines were incubated in phosphate-free RPMI-1640 medium (Life Technologies Inc.) for $6 \mathrm{~h}$, resuspended in retroviral supernatant supplemented with $8 \mu \mathrm{g} / \mathrm{ml}$ polybrene (Sigma-Aldrich, St. Louis, Missouri, USA), and then transferred to 24 -well plates. After centrifugation at $1,800 \mathrm{~g}$ for $30 \mathrm{~min}$, cells were incubated at $37^{\circ} \mathrm{C}$ overnight. The following day, the procedure was repeated. After transduction, cells were selected in $1.0 \mathrm{mg} / \mathrm{ml}$ of G418 (Geneticin; Life Technologies Inc.) for 12 days. Analysis of the inserted proviral genome by semiquantitative $P C R$. WASP exon 3 and 4 sequences were amplified from genomic DNA using sense $\left(5^{\prime}\right.$ GGA ACA GGA GCT GTA CTC AC- $\left.3^{\prime}\right)$ and antisense $\left(5^{\prime}\right.$-TCC ACT TTG CCT CTG ATT CC-3') primers and 30 cycles of amplification $\left(94^{\circ} \mathrm{C}\right.$ for $30 \mathrm{~s}, 60^{\circ} \mathrm{C}$ for $30 \mathrm{~s}$, and $72^{\circ} \mathrm{C}$ for $30 \mathrm{~s}$ ). The endogenous WASP gene fragment containing the intron 3 sequence $(100 \mathrm{bp})$ and the retroviral-derived integrants generate fragments of $272 \mathrm{bp}$ and $172 \mathrm{bp}$, respectively. Amplified products were separated on a $3 \%$ agarose gel and visualized by ethidium bromide staining. The intensity of each band was quantified

\section{Figure 2}

Flow-cytometric analysis of WASP. PBMCs were stained with mAbs for cell-surface antigens, fixed, and permeabilized. Cells were then reacted with anti-WASP $m A b$ (gray histogram) or a control Ab (white histogram) and further incubated with biotinconjugated anti-mouse IgG-1 followed by streptavidin-PE. Shown are the results of WASP expression in leukocytes from a healthy control and patients II-1 and II-2. using the BIT Image software (Aladdin Systems Inc., Watsonville, California, USA) and the NIH Image software. The ratio between the intensity of the endogenous WASP gene and the retroviral transgene products was then calculated as described (31).

TCR/CD3 downregulation assay. T cells $\left(10^{6}\right)$ were incubated with $1 \mu \mathrm{g} / \mathrm{ml}$ anti-CD3 mAb (Leu4; Becton Dickinson Immunocytometry Systems) for $30 \mathrm{~min}$ on ice. After washing, cells were reacted with $5 \mu \mathrm{g} / \mathrm{ml}$ biotinylated goat anti-mouse $\mathrm{Ab}$ (BioSource International, Camarillo, California, USA) for 30 min on ice and then warmed at $37^{\circ} \mathrm{C}$ for $60 \mathrm{~min}$ to allow downregulation of CD3. Cells were then transferred to ice, washed, and stained with PE-conjugated streptavidin (PharMingen). After fixation in $2 \%$ paraformaldehyde, CD3 expression was analyzed by FACS. Data were reported as the percentage of mean fluorescent intensity of control cells not subjected to incubation at $37^{\circ} \mathrm{C}(25)$. Analysis of differences among data groups was performed using the Student $t$ test for paired samples. $P$ values less than 0.05 were considered significant.

\section{Results}

Patients' immunophenotype and mosaic expression of WASP. The immunophenotypic characteristics of the patients' T lymphocytes are described in Table 1 . As part of the clinical evaluation of our WAS patients, we analyzed WASP expression in PBMCs by multicolor flow cytometry. As shown in Figure 2, total lymphocytes as well as T, $\mathrm{B}$, and NK cell subsets and $\mathrm{CD} 14^{+}$monocytes from a

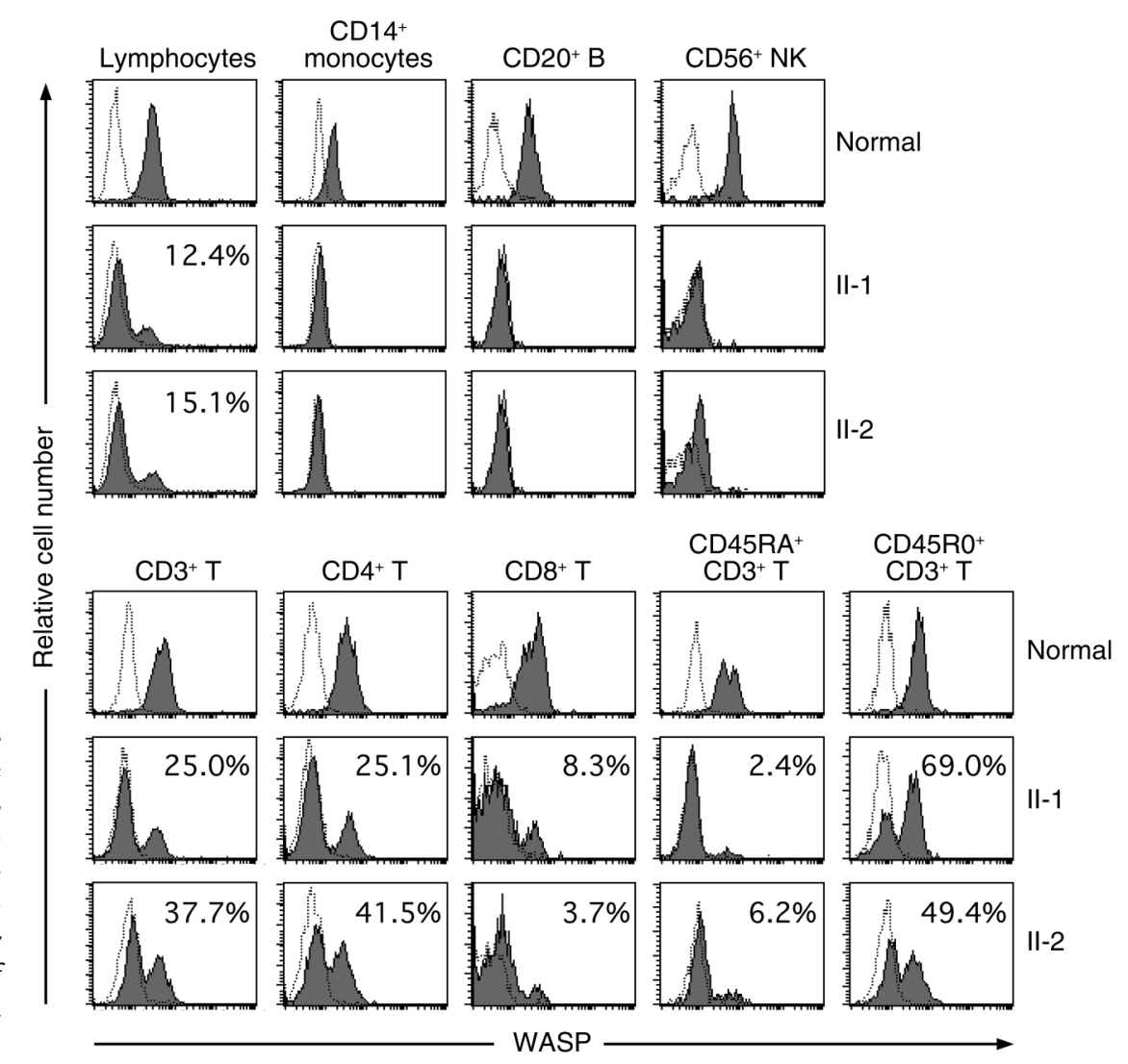


healthy control expressed WASP. In contrast, patients II- 1 and II-2 exhibited no detectable expression of WASP in $\mathrm{CD} 14^{+}$monocytes, but showed a sizable fraction of $\mathrm{WASP}^{+}$cells among their lymphocytes, suggesting the presence of somatic mosaicism. WASP ${ }^{+}$cells were found to include $\mathrm{CD}^{+} \mathrm{T}$ cells, but were not detected among $\mathrm{CD} 20^{+} \mathrm{B}$ cells and $\mathrm{CD} 56^{+} \mathrm{NK}$ cells in either patient. Both $\mathrm{CD}^{+}$and $\mathrm{CD}^{+} \mathrm{T}$ cells were involved in the mosaicism for WASP expression; however, $\mathrm{WASP}^{+}$cells were detected only in less than $10 \%$ of the patients' $\mathrm{CD}^{+} \mathrm{T}$ cells. Interestingly, more than half of $\mathrm{CD} 45 \mathrm{R}^{+} \mathrm{CD}^{+}$memory $\mathrm{T}$ lymphocytes expressed WASP in both patients, whereas WASP ${ }^{+}$cells were detected in only less than $10 \%$ of $\mathrm{CD} 45 \mathrm{RA}^{+} \mathrm{CD}^{+}$naive $\mathrm{T}$ cells.

Restoration of the WASP gene reading frame by a second-site mutation in revertant cells. The mutation causing WAS in this family was described previously as a single base $G$ insertion after nucleotide 1305 in exon 10 of the WASP gene (insG) (32). To determine whether the same mutation was present in $\mathrm{WASP}^{+} \mathrm{T}$ cells, direct sequence analysis was performed on genomic DNA obtained from sorted WASP ${ }^{+}$and WASP- $\mathrm{T}$ cells from patient II-1. As shown in Figure 3a, we found the insG mutation in DNA from the WASP- $T$ cells, whereas DNA from the WASP $^{+} \mathrm{T}$ cells showed a second-site mutation, represented by a 19 -bp deletion ( $\Delta 19 \mathrm{bp}$ ) encompassing the original ins $G$ mutation. When we analyzed the sequence of WASP exon 10 in subcloned PCR products obtained from unsorted cultured T lymphocytes of patient II-1, the $\Delta 19 \mathrm{bp}$ mutation was detected in $18.2 \%$ of 22 clones. In patient II-2, the coexistence of the $\Delta 19 \mathrm{bp}$ mutation was also demonstrated in DNA obtained from his
PBMCs (4.5\% of 22 clones). Sequence analysis of 100 normal chromosomes demonstrated the absence of the $\Delta 19 \mathrm{bp}$ mutation in the general population. Analysis of the DNA sequence surrounding the $\Delta 19 \mathrm{bp}$ mutation showed the presence of two 4-bp (CCTG) direct repeats and structural characteristics compatible with the formation of a hairpin loop containing five GC pairs within the deleted fragment (Figures 3 , $a$ and $b$ ).

Figure $3 \mathrm{c}$ shows the predicted structure of the mutated WASP molecules. The insG mutation causes a frameshift resulting in a novel sequence beginning at AA 424 and concluding in a premature termination at position 493. The $\Delta 19 \mathrm{bp}$ mutation leads to an internal deletion of 6-AA at the end of the proline-rich region, but to restoration of the WASP reading frame beginning at AA 427.

To determine the characteristics of WASP in the revertant cells, Western blot analysis was performed using the patients' cultured T lymphocytes. As shown in Figure 3d, T lymphocytes from a healthy control showed the presence of the expected approximately $59-\mathrm{kDa}$ WASP band. In contrast, lysates from the patients' lymphocytes showed a WASP band of lower molecular weight that was consistent with the predicted effects of the $\Delta 19 \mathrm{bp}$ mutation.

Genotypic analysis of lymphohematopoietic lineages. Purity of magnetic bead-isolated $\mathrm{CD}^{+} \mathrm{T}$ cells and $\mathrm{CD} 3$-depleted polymorphonuclear cells was more than $98 \%$, while sorted $\mathrm{CD} 20^{+} \mathrm{B}$ lymphocytes, CD56 ${ }^{+} \mathrm{NK}$ cells, and CD $14^{+}$monocytes contained less than $0.05 \%$ contaminating TCR- $\alpha \beta^{+} /$TCR- $\gamma \delta^{+}$cells (data not shown). Fluorescent genotyping of purified lymphocyte and myeloid cells showed clear evidence of the $\Delta 19 \mathrm{bp}$ mutation only

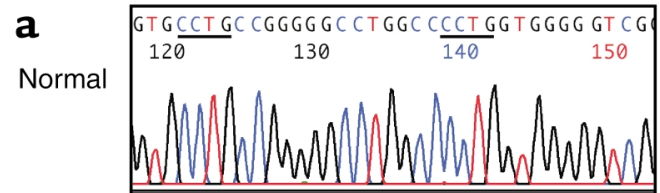

1305insG

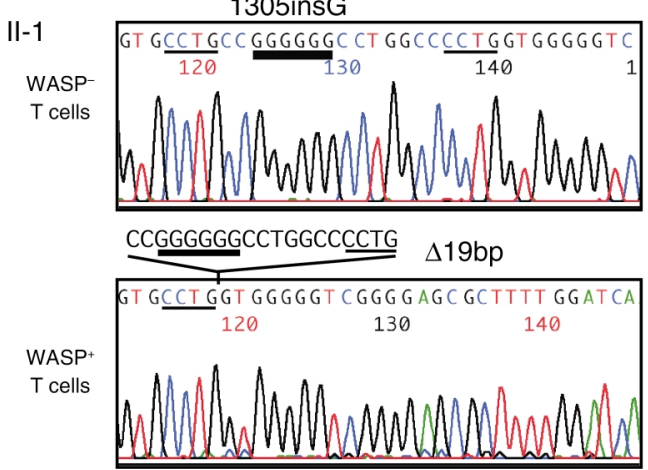

b

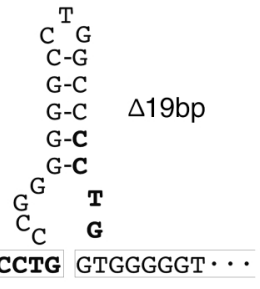

C
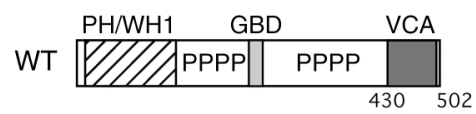

insG

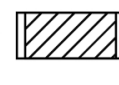

$\Delta 19 \mathrm{bp}$
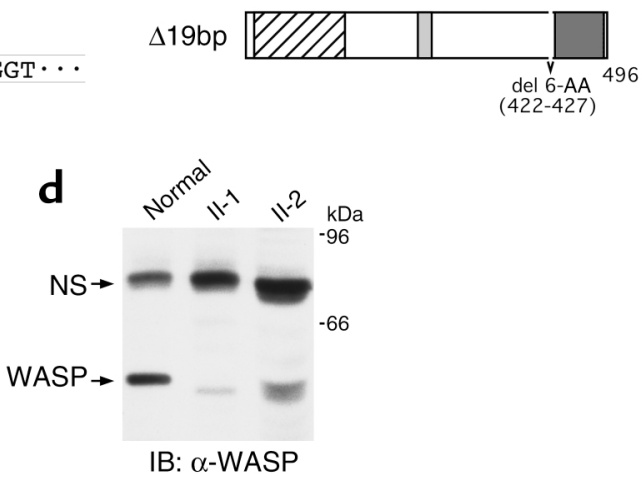

Figure 3

Characterization of WASP gene mutations and expression. (a) The WASP gene exon 10 sequence was amplified from DNA extracted from normal PBMCs, as well as WASP- T and WASP ${ }^{+}$T lymphocytes of patient II-1. Direct sequencing was performed using an automated sequencer. A thick bar highlights the position of the original insG mutation and thin bars show the 4-bp repeat sequences. (b) Model for the generation of hairpin structure facilitating the second-site mutation by slipped mispairing and deletion of $19 \mathrm{bp}$. (c) Predicted structures of mutated WASP molecules. PH, pleckstrin homology; WH, Wiskott homology; GBD, GTPase-binding domain; PPPP, proline rich. del, deletion. (d) Western blot analysis of WASP was performed using lysates of cultured T lymphocytes obtained from a healthy control and the patients. Arrows indicate the position of the WASP band and of a nonspecific protein reactive with the secondary Ab (NS). IB, immunoblotting. 
a
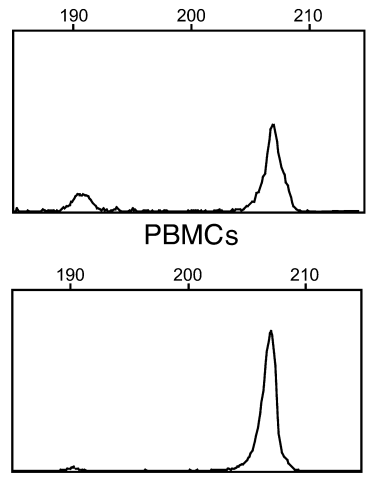

$\mathrm{CD}^{2} \mathrm{O}^{+} \mathrm{B}$ lymphocytes

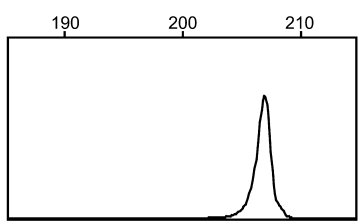

CD14 ${ }^{+}$monoocytes

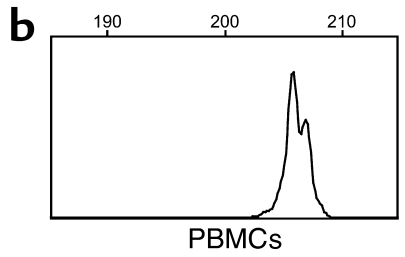

\section{Figure 4}

Genotypic analysis of the $\Delta 19 \mathrm{bp}$ second-site mutation. (a) GeneScan analysis of WASP exon 10 DNA sequence amplified from purified lymphocytic and myeloid cells of patient II-1. A peak of the size of approximately 207 nucleotides representing the insG mutation is evident in all cell types. A second peak of approximately 190 nucleotides generated by the $\Delta 19 \mathrm{bp}$ mutation is present in the samples amplified from PBMCs and purified CD3 ${ }^{+}$T lymphocytes. (b) Analysis of the WASP exon 10 DNA from WAS carrier subject $\mathrm{I}-1$ showed the presence of two overlapping peaks representing the normal and the insG-carrying alleles. The $\Delta 19 \mathrm{bp}$ mutation was undetectable in this sample.

among $\mathrm{CD}^{+} \mathrm{T}$ cells (Figure 4a). Genotyping and sequence analysis of DNA extracted from PBMCs of the patients' mother (subject I-1) showed that she was heterozygous carrier of the ins $\mathrm{G}$ mutation, but did not show detectable evidence of the $\Delta 19 \mathrm{bp}$ mutation (Figure $4 \mathrm{~b}$ and data not shown).

Policlonality of WASP ${ }^{+} T$ cells. High-resolution (Gene Scan) analysis of TCR- $\beta$ gene rearrangements showed a Gaussian-like distribution of the sizes of the TCR- $\beta$ amplificates in T lymphocytes from a healthy control and from sorted WASP- $T$ cells from patients II-1 and II-2 (Figure 5). TCR- $\beta$ rearrangements from the patients' WASP ${ }^{+}$cells were characterized by a lower number of peaks, suggesting that although policlonal, these cells carried a TCR repertoire of reduced complexity. As expected, leukemic MOLT- 4 cells showed a single peak indicative of monoclonal TCR- $\beta$ rearrangement (Figure 5).

Retrovirus-mediated expression of mutated WASP. To assess the expression and functional characteristics of the WASP mutants, retroviral vectors carrying wild-type
WASP cDNA, the ins $\mathrm{G}$, and the $\Delta 19 \mathrm{bp}$ mutants were used to transduce WAS $T$ cell lines carrying a null mutation of the WASP gene with no detectable WASP expression. As shown in Figure 6a, T cells untransduced or transduced with the Neo control vector did not express WASP, whereas WASP expression was well reconstituted in $T$ cells transduced with the vector carrying the wild-type WASP cDNA. T cells transduced with the insG mutant showed a very faint WASP band of lower molecular weight, as predicted by the early termination at codon 493. In contrast, $T$ cells transduced with the $\Delta 19 \mathrm{bp}$ mutant showed near normal levels of a WASP molecule with size characteristics compatible with the predicted internal deletion of 6 AA. As shown in Figure $6 \mathrm{~b}$, transgene-specific sequences were detected in T cells transduced with each of the retroviral vectors (wild type, insG, and $\Delta 19 \mathrm{bp}$ ), but not in untransduced $\mathrm{T}$ cells or cells transduced with the Neo control vector. Semiquantitative estimation of transgene copy number showed that all $\mathrm{T}$ cells transduced with the vectors carrying the WASP sequence had approximately one copy of the provirus per cell after G418 selection. These results indicate that low expression of WASP in T cells transduced with the insG mutation was neither due to low transduction efficiency nor to a failure of G418 selection, but more likely to instability of the WASP mRNA or protein carrying the insG mutation. Even when different anti-WASP Ab's were used for the detection of WASP (see Methods), T cells transduced with the insG mutant showed only minimal WASP expression of the mutated WASP (data not shown).

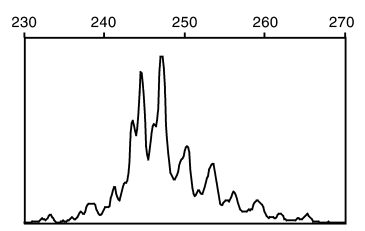

Peripheral T cells

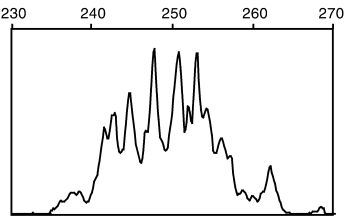

II-1 WASP-

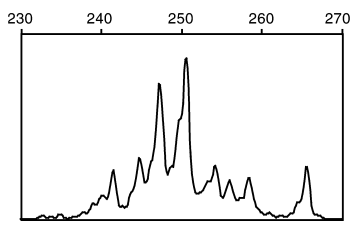

II-2 WASP-

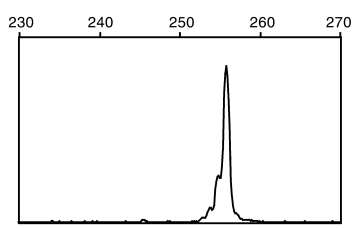

MOLT-4

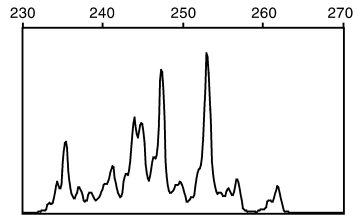

II-1 WASP+

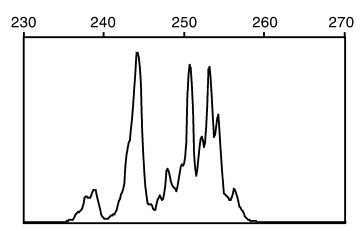

II-2 WASP $^{+}$

\section{Figure 5}

Analysis of TCR- $\beta$ gene rearrangements. Gaussian-like distribution of TCR- $\beta$ gene rearrangements observed in normal control CD $3^{+} \mathrm{T}$ lymphocytes and monoclonal rearrangement pattern detected in MOLT-4 leukemic cells are shown as controls for the results obtained from the study of TCR- $\beta$ gene rearrangements in WASP- ${ }^{-}$and WASP ${ }^{+}$ cells sorted from patients II- 1 and II-2. 

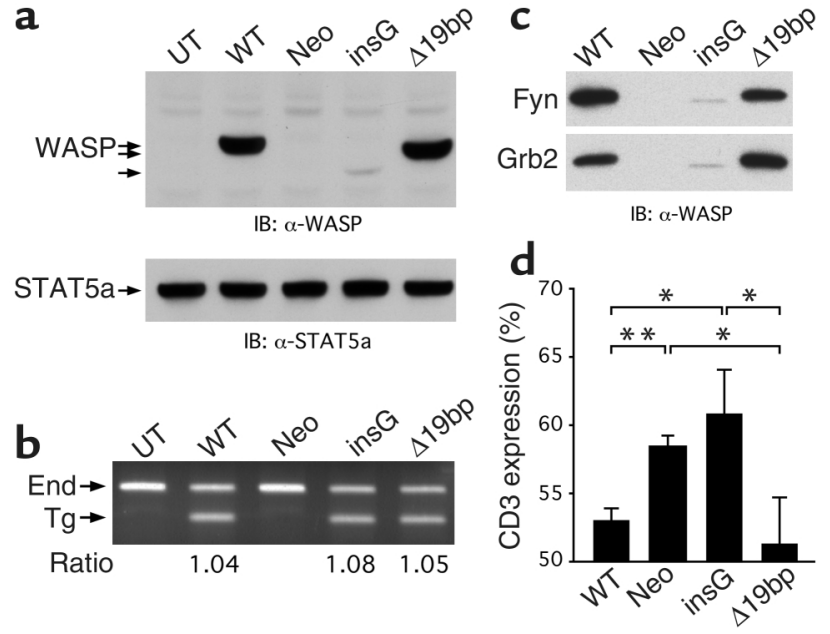

\begin{abstract}
Figure 6
In vitro reconstitution experiments. (a) Retrovirus-mediated expression of WASP. Western blot analysis of WASP was performed using lysates of WAS T cell lines transduced with the indicated retroviral vectors. Arrows indicate the position of the WASP bands. (b) Analysis of the inserted proviral genome by semiquantitative PCR. WASP exons 3 and 4 were amplified using DNA obtained from WAS T cell lines transduced with the indicated retroviral vector. The ratio of the band intensity of the endogenous WASP gene and the inserted transgene is shown. (c) In vitro binding of WASP to $\mathrm{SH} 3$ domain-containing proteins. Lysates were incubated and precipitated with $\mathrm{SH} 3-$ GST fusion proteins. Complexes were then resolved by SDS-PAGE, and WASP was detected by Western blot analysis. (d) Analysis of $\mathrm{TCR} / \mathrm{CD} 3$ downregulation. T cells were subjected to CD3 cross-linking at $37^{\circ} \mathrm{C}$ for $60 \mathrm{~min}$ and then stained with PE-conjugated streptavidin to analyze CD3 expression. Data are expressed as the percentage of mean fluorescent intensity of unstimulated control cells and represent the mean \pm SD of three independent experiments. ${ }^{*} P<0.05 ;{ }^{*} P<0.01$. UT, untransduced; Neo, Neo control vector; IB, immunoblotting; End, endogenous; Tg, transgene.
\end{abstract}

We next analyzed the binding ability of the mutant proteins to the SH3-domain containing proteins Fyn and Grb2. SH3 partners have been shown to interact with WASP through its proline-rich region (32), which is affected by the mutations found in these patients (Figure 3c). As expected and consistent with our previous experience (25), Fyn and Grb2 were able to bind in vitro to WASP from lysates of $T$ cells transduced with wild-type WASP cDNA, and no product was precipitable from lysates of cells transduced with the Neo control vector (Figure $6 \mathrm{c}$ ). In contrast, the amount of WASP precipitated by SH3-GST agarose beads was extremely low in lysates from $\mathrm{T}$ cells transduced with the insG mutant. Importantly, $\mathrm{T}$ cells transduced with the $\Delta 19 \mathrm{bp}$ mutant showed restored SH3-interaction comparable with the effect of wild-type WASP.

To further assess the functionality of the mutant proteins, we examined CD3-mediated downregulation of TCR/CD3 in transduced cells. As shown in Figure 6d and consistent with our previous results (25), the levels of TCR/CD3 expression decreased $1 \mathrm{~h}$ after simulation in T cells transduced with wild-type WASP cDNA and was impaired in T cells transduced with the Neo con- trol vector. Interestingly, the insG mutant failed to correct the downregulation defect, while $\mathrm{T}$ cells transduced with the $\Delta 19 \mathrm{bp}$ mutation showed significant improved responses to anti-CD3 stimulation.

Detection of circulating WASP' cells at various time points. To evaluate whether the accumulation of the $\mathrm{WASP}^{+} \mathrm{T}$ cells varied with time, a flow-cytometric analysis of WASP was performed using PBMCs of patient II-1 collected at 8, 11, and 18 years of age. As shown in Table 2, $\mathrm{WASP}^{+}$cells were detected in lymphocytes from all analyzed samples, and, importantly, the percentage of $\mathrm{WASP}^{+} \mathrm{T}$ cells was shown to increase with the age of the patient.

\section{Discussion}

Somatic revertant mosaicism due to back mutations or second-site mutations has been reported recently in several genetic diseases: tyrosinemia type 1 (6), Bloom syndrome (7), epidermolysis bullosa $(9,16)$, Fanconi anemia $(10,11,17)$, and primary immunodeficiencies, including adenosine deaminase deficiency $(12,13,18)$, $\mathrm{X}$-linked severe combined immunodeficiency (8), and WAS $(14,15)$. The molecular mechanism of such events remains mostly unclear, except for the few cases where intragenic recombination $(7,10)$, gene conversion (9), or DNA polymerase slippage $(11,15,18)$ has been shown. The real frequency of the occurrence of revertant mosaicism also remains to be determined, although it is assumed to be extremely low regardless of the mutagenic mechanism. Here we describe the unusual case of two WAS siblings presenting with somatic mosaicism due to the same second-site mutation, a deletion of 19 $\mathrm{bp}$, including the original single nucleotide insertion. The $\Delta 19 \mathrm{bp}$ mutation was observed only in the patients' $T$ lymphocytes and led to reconstitution of WASP expression. Sequencing analysis of the WASP gene in the general population excluded the possibility that the $\Delta 19$ bp mutation could be a functional polymorphism. The $\Delta 19 \mathrm{bp}$ mutation was not detectable in PBMC samples from the patients' mother, although somatic mosaicism would be difficult to detect in women due to

\section{Table 2}

Analysis of accumulation of WASP+ lymphocytes in patient II-1 with age

\begin{tabular}{|c|c|c|c|}
\hline Age $(y r)$ & $8.4^{\mathrm{A}}$ & $11.4^{\mathrm{A}}$ & 18.3 \\
\hline \multicolumn{4}{|c|}{ Absolute blood count $(/ \mu \mathrm{l})$} \\
\hline $\begin{array}{l}\text { WBCs } \\
\text { Lymphocytes }\end{array}$ & $\begin{array}{l}6,000 \\
1,440\end{array}$ & $\begin{array}{l}6,500 \\
1,430\end{array}$ & $\begin{array}{c}6,890 \\
703\end{array}$ \\
\hline \multicolumn{4}{|c|}{ T lymphocyte subsets (\%) } \\
\hline $\begin{array}{l}\mathrm{CD}^{+} \\
\mathrm{CD} 45 \mathrm{RO}^{-} \mathrm{CD}^{+} \\
\mathrm{CD} 45 \mathrm{RO}^{+} \mathrm{CD}^{+}\end{array}$ & $\begin{array}{l}63.8 \\
49.6 \\
14.2\end{array}$ & $\begin{array}{l}47.5 \\
27.5 \\
20.5\end{array}$ & $\begin{array}{l}63.9 \\
42.8 \\
21.1\end{array}$ \\
\hline \multicolumn{4}{|c|}{ WASP $^{+}$cells in lymphocyte subsets (\%) } \\
\hline $\begin{array}{l}\text { Lymphocytes } \\
\text { CD3 }{ }^{+} T \text { cells } \\
\text { CD45R0-CD3 }{ }^{+} T \text { cells } \\
\text { CD45R0 } 0^{+} \mathrm{CD} 3^{+} T \text { cells }\end{array}$ & $\begin{array}{c}3.6 \\
5.4 \\
0.9 \\
20.6\end{array}$ & $\begin{array}{c}7.5 \\
19.5 \\
1.4 \\
43.6\end{array}$ & $\begin{array}{c}12.4 \\
25.0 \\
2.1 \\
69.0\end{array}$ \\
\hline
\end{tabular}

AImmunophenotyping and flow cytometric analysis of WASP were performed on frozen samples. 
lack of selective advantage. Both patients, however, had the normal $46 \mathrm{XY}$ male karyotype, thus ruling out the possibility that the WASP ${ }^{+} \mathrm{T}$ lymphocytes could have arisen from maternal $\mathrm{T}$ cell engraftment. It is also extremely unlikely that both patients acquired the same mutant $\mathrm{T}$ cells postnatally from infusions of blood products. We therefore conclude that the $\mathrm{T}$ lymphocytes carrying the $\Delta 19 \mathrm{bp}$ mutation originated in both patients in vivo due to a second-site mutation.

Revertant mosaicism due to a second-site mutation has been reported previously (16-18). In particular, the occurrence of the same second-site mutation in two brothers with Fanconi anemia has been reported by Waisfisz et al. (17), who proposed the presence of the same second-site mutation having resulted from the same specific molecular mechanism in the two subjects. Similarly, our observations strongly suggest that a common underlying mechanism resulted in the occurrence of the same second-site mutation in the two brothers with WAS, described here. Slipped mispairing is a wellaccepted mechanism for genomic instability, resulting in insertions and deletions of repetitive sequences in many genetic disorders (19-21). Analysis of the sequence surrounding the $\Delta 19 \mathrm{bp}$ second-site mutation (Figure $3 \mathrm{c}$ ) leads us to propose that slipped mispairing is at the basis of our findings. We argue that the presence of two 4-bp direct sequences (CCTG) and the hairpin structure created by five GC pairs within the deleted fragment are able to induce slippage and mispairing of the nascent and template strands during DNA replication.

The slipped mispairing event leading to the $\Delta 19 \mathrm{bp}$ deletion may have occurred in a primitive pluripotent hematopoietic progenitor or in a committed $\mathrm{T}$ cell precursor. The latter possibility is supported by the notion that WAS-carrier women preferentially inactivate the $\mathrm{X}$ chromosome carrying the mutated WASP gene in hematopoietic progenitors $(33,34)$ and all mature hematopoietic cells $(35,36)$. WASP ${ }^{+}$cells deriving from a revertant pluripotent hematopoietic progenitor would therefore be expected to show selective advantage among all lineages, whereas we could only detect WASP ${ }^{+}$cells within the $\mathrm{CD}^{+} / \mathrm{CD}^{+}$and $\mathrm{CD}^{+} / \mathrm{CD}^{+} \mathrm{T}$ lymphocytes from our two patients.

On the other hand, the occurrence of the revertant mosaicism in both patients suggests that the secondsite mutation is not an extremely rare event. It seems unlikely, therefore, that such occurrence would coincidentally target only the same cell-differentiation stage in both patients. On the contrary, slipped mispairing could be a relatively common event with respect to nucleotide $1299-1316$ of the WASP sequence and, therefore, that the $\Delta 19 \mathrm{bp}$ deletion may occur in a variety of cell types, but become apparent only in cells characterized by a long life span that can accumulate over time. To the detection limits of our genotyping assay, we could clearly demonstrate the presence of the $\Delta 19 \mathrm{bp}$ deletion only among patients' purified $\mathrm{T}$ lymphocytes, however. These findings, taken together with the detection of WASP ${ }^{+}$cells among both $\mathrm{CD}^{+}$and $\mathrm{CD}^{+}{ }^{+} \mathrm{Tlym}-$ phocytes and the observation of polyclonal TCR- $\beta$ gene rearrangements in $\mathrm{WASP}^{+} \mathrm{T}$ cells, allow placement of the second-site mutation event at the level of a $T$ cell progenitor cell before $\mathrm{CD} 4 / \mathrm{CD} 8$ lineage commitment and TCR rearrangement.

Consistent with our previous observations (15), there is evidence of in vivo selective advantage of WASP $^{+} \mathrm{T}$ lymphocytes in these two patients. Flow-cytometric studies showed that $2.4-6.2 \% \mathrm{WASP}^{+}$cells appeared

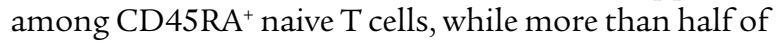
the CD45R0 $0^{+}$memory $\mathrm{T}$ cells were $\mathrm{WASP}^{+}$in both patients. These findings clearly indicate WASP ${ }^{+} \mathrm{T}$ cells had selective growth advantage over WASP- counterparts during the differentiation and proliferation steps leading to the acquisition of the immunological memory. The reasons why the patients showed higher proportion of revertant $\mathrm{CD}^{+} \mathrm{T}$ lymphocytes than $\mathrm{CD}^{+} \mathrm{T}$ cells are presently unclear. Immunophenotyping of $\mathrm{T}$ lymphocyte subsets demonstrated an increased number of activated memory $\mathrm{CD} 4^{+} \mathrm{T}$ lymphocytes in both patients (Table 1). It is therefore possible that peripheral expansion of these $\mathrm{CD}^{+} \mathrm{T}$ lymphocytes had favored the selective advantage of revertant cells within this subset of lymphocytes.

Our data also suggest that the expression of the internally deleted WASP mutant generated by the $\Delta 19 \mathrm{bp}$ mutation was responsible for the in vivo selective advantage of revertant $T$ cells through reconstitution of WASP function. It has been recognized recently that WASP function is regulated through an autoinhibitory conformation involving intramolecular interaction between the GTPase-binding domain and the verprolin homology, cofilin homology, and acidic (VCA) region of WASP (37). The original insG mutation destroys the VCA region, thus likely interfering with the protein tertiary structure and resulting in instability. On the contrary, the $\Delta 19 \mathrm{bp}$ mutation results in the deletion of 6-AA at the end of the proline-rich region but restores the sequence of the VCA domain, which can regenerate its ability to interact to the GTPase-binding domain, thus resulting in a more stable protein (38). Accordingly, our in vitro reconstitution experiments showed that WASP expression, binding to SH3 partners, and TCR/CD3 downregulation in T cells carrying the $\Delta 19 \mathrm{bp}$ mutant were compatible with those observed in the presence of wild-type WASP and indicated that the $\Delta 19 \mathrm{bp}$ mutant is functional.

The availability of archived PBMC samples from patient II-1 offered us a unique opportunity to study the accumulation of WASP ${ }^{+} \mathrm{T}$ lymphocytes over time in this patient. We were able to detect WASP expression in his lymphocytes at age 8 , and the percentage of WASP ${ }^{+}$ cells increased with age, suggesting that the accumulation of WASP ${ }^{+}$cells in peripheral blood had started more than 10 years ago. Neither of the two patients in this study, however, have yet shown improvement of their clinical phenotype. This is in contrast to a previously described case of somatic mosaicism in a WAS patient carrying more than $80 \%$ of revertant $\mathrm{T}$ lymphocytes (15), but is consistent with the finding of Ariga et al. 
who did not observe clinical improvement in another case of WAS with revertant mosaicism (14). One explanation for the lack of clinical benefit from the occurrence of the second-site mutation in the patients described here is that the absolute numbers of $\mathrm{WASP}^{+} \mathrm{T}$ lymphocytes, and especially revertant naive $\mathrm{T}$ lymphocytes, were simply not high enough to correct the WAS phenotype. If this is the case, it is possible that an improvement will be observed in the future because of the selective accumulation of WASP ${ }^{+} \mathrm{T}$ lymphocytes. Alternatively, the function of the mutant WASP carrying the $\Delta 19 \mathrm{bp}$ mutation may not be as effective in vivo as the wild-type WASP.

In summary, our studies provide evidence for potential beneficial outcomes of slipped mispairing events and tie this genetic mechanism to the generation of somatic mosaicism. Although our current knowledge regards back mutations or second-site mutations as extremely rare events, the observation of the present two cases raises the possibility that genetic reversions may take place more often than is commonly accepted, but remain undetected because they do not necessarily result in modification of the clinical phenotype. Because revertant mosaicism is a natural form of gene therapy, the identification of revertants and characterization of mechanism underlying back mutations or second-site mutations is obviously of great importance for the development of new therapeutic strategies for genetic disorders and deserve active investigation.

\section{Acknowledgments}

The authors are thankful to R. Michael Blaese for providing historical samples from patient II-1 and to Andy Baxevanis for help with sequence analysis. We also thank Jacqueline Keller for secretarial assistance. This work was supported in part by Japan Society for the Promotion of Science Research Fellowships for Japanese Biomedical and Behavioral Researchers at the NIH.

1. Ochs, H.D. 1998. The Wiskott-Aldrich syndrome. Springer Semin. Immunopathol. 19:435-458.

2. Derry, J.M., Ochs, H.D., and Francke, U. 1994. Isolation of a novel gene mutated in Wiskott-Aldrich syndrome. Cell. 78:635-644.

3. Stewart, D.M., et al. 1996. Studies of the expression of the Wiskott-Aldrich syndrome protein. J. Clin. Invest. 97:2627-2634.

4. Parolini, O., et al. 1997. Expression of Wiskott-Aldrich syndrome protein (WASP) gene during hematopoietic differentiation. Blood. 90:70-75.

5. Snapper, S.B., and Rosen, F.S. 1999. The Wiskott-Aldrich syndrome protein (WASP): roles in signaling and cytoskeletal organization. Annu. Rev. Immunol. 17:905-929.

6. Kvittingen, E.A., Rootwelt, H., Berger, R., and Brandtzaeg, P. 1994. Selfinduced correction of the genetic defect in tyrosinemia type I.J. Clin. Invest. 94:1657-1661.

7. Ellis, N.A., et al. 1995. Somatic intragenic recombination within the mutated locus BLM can correct the high sister-chromatid exchange phenotype of Bloom syndrome cells. Am. J. Hum. Genet. 57:1019-1027.

8. Stephan, V., et al. 1996. Atypical X-linked severe combined immunodeficiency due to possible spontaneous reversion of the genetic defect in T cells. N. Engl. J. Med. 335:1563-1567.

9. Jonkman, M.F., et al. 1997. Revertant mosaicism in epidermolysis bullosa caused by mitotic gene conversion. Cell. 88:543-551.

10. Lo Ten Foe, J.R., et al. 1997. Somatic mosaicism in Fanconi anemia: molecular basis and clinical significance. Eur.J. Hum. Genet. 5:137-148.

11. Gregory, J.J., Jr., et al. 2001. Somatic mosaicism in Fanconi anemia: evidence of genotypic reversion in lymphohematopoietic stem cells. Proc. Natl.
Acad. Sci. U. S. A. 98:2532-2537.

12. Hirschhorn, R., et al. 1996. Spontaneous in vivo reversion to normal of an inherited mutation in a patient with adenosine deaminase deficiency. Nat. Genet. 13:290-295.

13. Ariga, T., et al. 2001. T-cell lines from 2 patients with adenosine deaminase (ADA) deficiency showed the restoration of ADA activity resulted from the reversion of an inherited mutation. Blood. 97:2896-2899.

14. Ariga, T., et al. 2001. Spontaneous in vivo reversion of an inherited mutation in the Wiskott-Aldrich syndrome. J. Immunol. 166:5245-5249.

15. Wada, T., et al. 2001. Somatic mosaicism in Wiskott-Aldrich syndrome suggests in vivo reversion by a DNA slippage mechanism. Proc. Natl. Acad. Sci. U. S. A. 98:8697-8702.

16. Darling, T.N., Yee, C., Bauer, J.W., Hintner, H., and Yancey, K.B. 1999. Revertant mosaicism: partial correction of a germ-line mutation in COL17A1 by a frame-restoring mutation. J. Clin. Invest. 103:1371-1377.

17. Waisfisz, Q., et al. 1999. Spontaneous functional correction of homozygous Fanconi anaemia alleles reveals novel mechanistic basis for reverse mosaicism. Nat. Genet. 22:379-383.

18. Arredondo-Vega, F.X., et al. 2002. Adenosine deaminase deficiency with mosaicism for a "second-site suppressor" of a splicing mutation: decline in revertant T lymphocytes during enzyme replacement therapy. Blood. 99:1005-1013.

19. Levinson, G., and Gutman, G.A. 1987. Slipped-strand mispairing: a major mechanism for DNA sequence evolution. Mol. Biol. Evol. 4:203-221.

20. Darvasi, A., and Kerem, B. 1995. Deletion and insertion mutations in short tandem repeats in the coding regions of human genes. Eur. J. Hum. Genet. 3:14-20.

21. Oron-Karni, V., Filon, D., Rund, D., and Oppenheim, A. 1997. A novel mechanism generating short deletion/insertions following slippage is suggested by a mutation in the human alpha2-globin gene. Hum. Mol. Genet. 6:881-885.

22. Bzymek, M., and Lovett, S.T. 2001. Instability of repetitive DNA sequences: the role of replication in multiple mechanisms. Proc. Natl. Acad. Sci.U. S. A. 98:8319-8325.

23. Pearson, C.E., and Sinden, R.R. 1998. Trinucleotide repeat DNA structures: dynamic mutations from dynamic DNA. Curr. Opin. Struct. Biol. 8:321-330.

24. Zhu, Q., et al. 1995. The Wiskott-Aldrich syndrome and X-linked congenital thrombocytopenia are caused by mutations of the same gene. Blood. 86:3797-3804.

25. Wada, T., Jagadeesh, G.J., Nelson, D.L., and Candotti, F. 2002. Retrovirusmediated WASP gene transfer corrects Wiskott-Aldrich syndrome T cell dysfunction. Hum. Gene Ther. 13:1039-1046.

26. Candotti, F., et al. 1999. Retrovirus-mediated WASP gene transfer corrects defective actin polymerization in B cell lines from Wiskott-Aldrich syndrome patients carrying 'null' mutations. Gene Ther. 6:1170-1174.

27. Candotti, F., et al. 1997. Structural and functional basis for JAK3-deficient severe combined immunodeficiency. Blood. 90:3996-4003.

28. Assaf, C., et al. 2000. High detection rate of T-cell receptor beta chain rearrangements in T-cell lymphoproliferations by family specific polymerase chain reaction in combination with the GeneScan technique and DNA sequencing. Blood. 96:640-646.

29. Miller, A.D., et al. 1991. Construction and properties of retrovirus packaging cells based on gibbon ape leukemia virus. J. Virol. 65:2220-2224.

30. Treisman, J., et al. 1995. Interleukin-2-transduced lymphocytes grow in an autocrine fashion and remain responsive to antigen. Blood. 85:139-145.

31. Onodera, M., et al. 1998. Development of improved adenosine deaminase retroviral vectors. J. Virol. 72:1769-1774.

32. Kolluri, R., et al. 1995. Identification of WASP mutations in patients with Wiskott-Aldrich syndrome and isolated thrombocytopenia reveals allelic heterogeneity at the WAS locus. Hum. Mol. Genet. 4:1119-1126.

33. Mantuano, E., et al. 1993. Analysis of X-chromosome inactivation in bone marrow precursors from carriers of Wiskott-Aldrich syndrome and $\mathrm{X}$-linked severe combined immunodeficiency: evidence that the WiskottAldrich gene is expressed prior to granulocyte-macrophage colony-forming-unit. Immunodeficiency. 4:271-276.

34. Wengler, G., Gorlin, J.B., Williamson, J.M., Rosen, F.S., and Bing, D.H. 1995. Nonrandom inactivation of the $\mathrm{X}$ chromosome in early lineage hematopoietic cells in carriers of Wiskott-Aldrich syndrome. Blood. 85:2471-2477.

35. Fearon, E.R., Kohn, D.B., Winkelstein, J.A., Vogelstein, B., and Blaese, R.M. 1988. Carrier detection in the Wiskott-Aldrich syndrome. Blood. 72:1735-1739.

36. Greer, W.L., et al. 1989. X-chromosome inactivation in the Wiskott-Aldrich syndrome: a marker for detection of the carrier state and identification of cell lineages expressing the gene defect. Genomics. 4:60-67.

37. Kim, A.S., Kakalis, L.T., Abdul-Manan, N., Liu, G.A., and Rosen, M.K. 2000. Autoinhibition and activation mechanisms of the Wiskott-Aldrich syndrome protein. Nature. 404:151-158.

38. Devriendt, K.J., et al. 2001. Constitutively activating mutation in WASP causes X-linked severe congenital neutropenia. Nat. Genet. 27:313-317. 